\title{
A distortion theorem \\ for quasiconformal automorphisms of the unit disk
}

\author{
by DARIUsz PARTYKa (Lublin)
}

\begin{abstract}
We give a distortion theorem for quasiconformal automorphisms of the unit disk and its application to improving some results due to Douady and Earle.

1. Introduction. In various estimates connected with the conformally natural quasiconformal extension of a quasisymmetric automorphism $f$ of the unit circle $T$, as given by Douady and Earle [3], the following distortion problem plays an important role. If $f$ is a $K$-quasiconformal self-mapping of the unit disk normalized by the condition $\int_{T} f(z)|d z|=0$, find an estimate $r(K)$ of $|f(0)|$. We were able to find an estimate $r(K)$ such that $r(K) \rightarrow 0$ as $K$ tends to 1 . Consequently, in a simple way, some results due to Douady and Earle [3] could be improved for $K$ near 1 .
\end{abstract}

2. Let $T$ be the unit circle. We start with the following

LEMMA. If $f$ is a $K$-quasiconformal self-mapping of the unit disk $D$ and

$$
\int_{T} f(z)|d z|=0
$$

then

(2) $\quad|f(0)| \leq 2 p(K)=\frac{8}{\pi(K+1)}\left(K 2^{7(1-1 / K) / 2}-2^{7(1-K) / 2}\right)$

for $1 \leq K \leq 1.044$ and

$$
|f(0)| \leq 1-2\left(1+\sqrt{3\left(4^{3 K-2}-1\right)}\right)^{-1}
$$

for other $K$.

1991 Mathematics Subject Classification: Primary 30C60.

Key words and phrases: quasiconformal mappings, distortion theorems, Möbius transformations, quasiconformal extensions. 
Proof. Using the fact that for every arc $I \subset T$ of length $|I|=\frac{2}{3} \pi$ we have, by (1), $|f(I)| \leq \frac{4}{3} \pi$ (cf. [5]), and from the quasi-invariance of the harmonic measure [4], we get the estimate

$$
|f(0)| \leq \frac{1}{2}+\frac{\sqrt{3}}{2} \cot \left(\frac{\pi}{3}+\arccos \Phi_{K}\left(\frac{\sqrt{3}}{2}\right)\right),
$$

where $\Phi_{K}=\mu^{-1}\left(K^{-1} \mu\right)$ and $\mu(r), 0<r<1$, is the module of $D$ slit from 0 to $r$ along the positive real axis [6]. For details cf. [5]. Since $\Phi_{K}^{2}(r)+$ $\Phi_{1 / K}^{2}\left(\sqrt{1-r^{2}}\right)=1$ for $K>0$ and $0 \leq r \leq 1$, as shown in [1], from the inequality [6]

$$
\Phi_{K}(r) \leq 4^{1-1 / K} r^{1 / K}, \quad K \geq 1,0 \leq r \leq 1,
$$

we derive (3) after suitable transformations of (4) for every $K \geq 1$. But (3) is not sharp because its r.h.s. tends to $1 / 2$ as $K \rightarrow 1$. In what follows we are going to replace the r.h.s. in (3) so as to obtain an asymptotically sharp estimate.

Let

$$
h_{a}(z)=\frac{z-a}{1-\bar{a} z}, \quad z, a \in D,
$$

be a Möbius transformation of $D$. Assume $f(0)=a$. By the Darboux property there exist $z_{1}, z_{2} \in T$ such that $h_{a} \circ f\left(z_{1}\right)=z_{2}$ and $h_{a} \circ f\left(-z_{1}\right)=$ $-z_{2}$. Then the function $\bar{z}_{2} h_{a} \circ f\left(z_{1} z\right)$ is a $K$-quasiconformal self-mapping of $D$ which keeps the points $-1,0,1$ fixed, and due to its Hölder continuity [6], we have

$$
\begin{aligned}
& 16^{1-K}|z-1|^{K} \leq\left|\bar{z}_{2} h_{a} \circ f\left(z_{1} z\right)-1\right| \leq 16^{1-1 / K}|z-1|^{1 / K} \\
& 16^{1-K}|z+1|^{K} \leq\left|\bar{z}_{2} h_{a} \circ f\left(z_{1} z\right)+1\right| \leq 16^{1-1 / K}|z+1|^{1 / K}
\end{aligned}
$$

for every $z \in T$. Hence

$$
\text { (5) } \begin{aligned}
& \frac{1}{2 \pi} \int_{T}\left|h_{a} \circ f(z)-z_{2} \bar{z}_{1} z\right||d z| \\
\leq & \frac{4}{2 \pi} \int_{0}^{\pi / 2}\left(2^{1 / K} 16^{1-1 / K}\left(\sin \frac{t}{2}\right)^{1 / K} \cos \frac{t}{2}-2^{K} 16^{1-K}\left(\sin \frac{t}{2}\right)^{K} \cos \frac{t}{2}\right) d t \\
= & \frac{4}{\pi(K+1)}\left(K 2^{7(1-1 / K) / 2}-2^{7(1-K) / 2}\right)=p(K) .
\end{aligned}
$$

From this and (1) we get

$$
|a|=\frac{1}{2 \pi}\left|\int_{T} h_{-a}\left(z_{2} \bar{z}_{1} z\right)\right| d z\left|-\int_{T} h_{-a}\left(h_{a} \circ f(z)\right)\right| d z||
$$




$$
=\frac{1}{2 \pi}\left|\int_{T} \frac{\left(1-|a|^{2}\right)\left(z_{2} \bar{z}_{1} z-h_{a} \circ f(z)\right)}{\left(1+\bar{a} z_{2} \bar{z}_{1} z\right)\left(1+\bar{a} h_{a} \circ f(z)\right)}\right| d z|| \leq \frac{1+|a|}{1-|a|} p(K),
$$

which implies, in view of (3),

$$
|a| \leq \frac{1-p(K)-\sqrt{(1-p(K))^{2}-4 p(K)}}{2} \leq 2 p(K)<\frac{1}{3},
$$

since $1 \leq K \leq 1.044$, and this ends the proof.

3. For any automorphism $\gamma$ of $T$, as shown by Choquet [2], the mapping

$$
D \ni w \mapsto \frac{1}{2 \pi} \int_{T} h_{z} \circ \gamma(u) \operatorname{Re} \frac{u+w}{u-w}|d u| \in D
$$

is an automorphism of $D$ for any fixed $z \in D$. Therefore the equation

$$
\frac{1}{2 \pi} \int_{T} h_{z} \circ \gamma(u) \operatorname{Re} \frac{u+w}{u-w}|d u|=0
$$

implicitly defines a function $w=F_{\gamma}(z)$. It is quite easy to show that $F_{\gamma}$ is a real-analytic diffeomorphic self-mapping of $D$ which has a continuous extension to an automorphism $\gamma^{-1}$ of $T$, and for any Möbius transformations $\eta_{1}, \eta_{2}$

$$
F_{\eta_{1} \circ \gamma \circ \eta_{2}}=\eta_{2}^{-1} \circ F_{\gamma} \circ \eta_{1}^{-1} \text {. }
$$

For details see [5]. As a matter of fact, $F_{\gamma}^{-1}$ coincides with the mapping $E(\gamma)$ found by Douady and Earle [3; Theorem 1], but the construction of $F_{\gamma}$ is much simpler as compared with that of $E(\gamma)$.

Assume that $\gamma$ admits a $K$-quasiconformal extension $f$ to $D$. We shall apply the lemma to estimate the complex dilatation of $F_{\gamma}$ in $D$ for small $K$. In view of (7) it is sufficient to do this at the point 0 in the case when $F_{\gamma}(0)=0$. Then by $(6)$

$$
\int_{T} f(u)|d u|=0
$$

and differentiating both sides of (6) at 0 with respect to $z$ and $\bar{z}$ we obtain

$$
\partial_{z} F_{\gamma}(0)=\frac{\bar{A}-\bar{C} B}{|A|^{2}-|B|^{2}}, \quad \partial_{\bar{z}} F_{\gamma}(0)=\frac{\bar{A} C-B}{|A|^{2}-|B|^{2}},
$$

where

$$
A=\frac{1}{2 \pi} \int_{T} \bar{u} f(u)|d u|, \quad B=\frac{1}{2 \pi} \int_{T} u f(u)|d u|, \quad C=-\frac{1}{2 \pi} \int_{T} f^{2}(u)|d u| .
$$

Let $a=f(0)$ and let $z_{1}, z_{2}$ be as in the proof of the lemma. Since $\mid h_{a}(u)-$ $u|\leq 2| a \mid$ for every $u \in T$, by the lemma and (5) we obtain for $K \leq 1.044$ 
the following estimates:

$$
\begin{aligned}
1-|A| & \leq \frac{1}{2 \pi}\left|\int_{T} z_{2} \bar{z}_{1} u \bar{u}\right| d u\left|-\int_{T} \bar{u} f(u)\right| d u|| \\
& \leq \frac{1}{2 \pi} \int_{T}\left|z_{2} \bar{z}_{1} u-h_{a} \circ f(u)\right||d u|+\frac{1}{2 \pi} \int_{T}\left|h_{a} \circ f(u)-f(u)\right||d u| \\
& \leq p(K)+2|a|=5 p(K), \\
|B| & =\frac{1}{2 \pi}\left|\int_{T} u f(u)\right| d u\left|-\int_{T} z_{2} \bar{z}_{1} u^{2}\right| d u|| \\
& \leq \frac{1}{2 \pi} \int_{T}\left|f(u)-h_{a} \circ f(u)\right||d u|+\frac{1}{2 \pi} \int_{T}\left|h_{a} \circ f(u)-z_{2} \bar{z}_{1} u\right||d u| \\
& \leq p(K)+2|a|=5 p(K), \\
|C| & =\frac{1}{2 \pi}\left|\int_{T}\left(z_{2} \bar{z}_{1}\right)^{2} u^{2}\right| d u\left|-\int_{T} f^{2}(u)\right| d u|| \\
& \leq \frac{2}{2 \pi} \int_{T}\left|z_{2} \bar{z}_{1} u-h_{a} \circ f(u)\right||d u|+\frac{2}{2 \pi} \int_{T}\left|h_{a} \circ f(u)-f(u)\right||d u| \\
& \leq 2 p(K)+4|a| \leq 10 p(K) .
\end{aligned}
$$

Hence

$$
\begin{aligned}
\left|\frac{\partial_{\bar{z}} F_{\gamma}(0)}{\partial_{z} F_{\gamma}(0)}\right| & =\left|\frac{\bar{A} C-B}{\bar{A}-\bar{C} B}\right| \leq \frac{|C|+|B|}{1-|1-A|-|C||B|} \\
& \leq \frac{15 p(K)}{1-5 p(K)-50 p^{2}(K)}<1
\end{aligned}
$$

if $K \leq 1.01$.

Thus, in a simple and short way we have obtained the

THEOREM. If an automorphism $\gamma$ of $T$ admits a $K$-quasiconformal extension to $D$, where $1 \leq K \leq 1.01$, then the mappings $F_{\gamma}$ and $E(\gamma)$ are $K^{*}$-quasiconformal, where

$$
K^{*} \leq 1+30 p(K)(1+116 p(K))
$$

What is important, we have obtained an explicit estimate which tends to 1 as $K \rightarrow 1$. In this sense the above theorem improves the results found by Douady and Earle [3; Corollary 2, Proposition 7]. Using the theory of Teichmüller mappings they have proved that, given $\epsilon>0$, there exists $\delta>0$ such that $K^{*} \leq K^{3+\epsilon}$ if $K \leq 1+\delta$ [3; Corollary 2]. Their explicit estimate [3; Proposition 7] starts from $4 \cdot 10^{8} e^{35}$ for $K$ near 1 . 


\section{References}

[1] G. D. Anderson, M. K. Vamananmurthy and M. Vuorinen, Distortion functions for plane quasiconformal mappings, Israel J. Math. 62 (1) (1988), 1-16.

[2] G. Choquet, Sur un type de transformation analytique généralisant la représentation conforme et définie au moyen de fonctions harmoniques, Bull. Sci. Math. (2) 69 (1945), 156-165.

[3] A. Douady and C. J. Earle, Conformally natural extension of homeomorphisms of the circle, Acta Math. 157 (1-2) (1986), 23-48.

[4] J. Hersch, Longueurs extrémales, mesure harmonique et distance hyperbolique, C. R. Acad. Sci. Paris 235 (1952), 569-571.

[5] A. Lecko and D. Partyka, An alternative proof of a result due to Douady and Earle, Ann. Univ. Maria Curie-Skłodowska Sect. A 42 (1988), 59-68.

[6] O. Lehto and K. I. Virtanen, Quasiconformal Mappings in the Plane, Springer, Berlin 1973.

INSTITUTE OF MATHEMATICS

M. CURIE-SKŁODOWSKA UNIVERSITY

PL. MARII CURIE-SKLODOWSKIEJ 1

20-031 LUBLIN, POLAND 